\title{
PLANEJAMENTO AMOSTRAL DA PRESSÃo DE PRECONSOLIDAÇÃO DE UM LATOSSOLO VERMELHO DISTROFÉRRICO(1)
}

\author{
Ivoney Gontijo ${ }^{(2)}$, Moacir de Souza Dias Junior ${ }^{(3)}$, Marcelo Silva de \\ Oliveira $^{(4)}$, Cezar Francisco Araujo Junior ${ }^{(5)}$, Bruno Silva Pires ${ }^{(5)}$ \& \\ Carloeme Alves de Oliveira ${ }^{(6)}$
}

\begin{abstract}
RESUMO
Atualmente, a pressão de preconsolidação $\left(\sigma_{p}\right)$ tem sido utilizada como uma ferramenta no estudo do processo de compactação do solo. O objetivo deste trabalho foi determinar a configuração e o número adequado de amostras para determinação da $\sigma_{\mathrm{p}}$ em um Latossolo Vermelho distroférrico, bem como caracterizar sua variabilidade e distribuição espacial, utilizando métodos da Estatística Clássica e da Geoestatística. O estudo foi realizado em uma área experimental, no município de Lavras-MG, sob plantio convencional nos últimos 30 anos, no qual são realizados anualmente uma aração e duas gradagens. A área experimental possui dimensões de $32 \times 160 \mathrm{~m}\left(5.120 \mathrm{~m}^{2}\right)$, com 68 pontos distanciados a $10 \mathrm{~m}$ no eixo $X$ e a $8 \mathrm{~m}$ no eixo $Y$, mais seis transectos, sendo quatro com pontos distanciados de $2 \mathrm{~m}$ e dois com pontos distanciados de $1 \mathrm{~m}$, visando detectar variações a pequenas distâncias. Foram coletadas 98 amostras indeformadas na profundidade de 0-3 cm, as quais foram submetidas ao ensaio de compressão uniaxial, para obtenção das $\sigma_{\mathrm{p}}$. Por meio dos parâmetros da Estatística Clássica, estabeleceu-se o número adequado de amostras para determinar a $\sigma_{\mathrm{p}}$, que foi igual a 10 pontos. A maior variabilidade foi obtida para a $\sigma_{p}(C V 14,8 \%)$, e a menor, para a umidade do solo (CV 12,0\%). Ambas as variáveis apresentaram distribuição normal, com modelo esférico ajustado, e moderada estrutura de dependência espacial, com alcance de 19,5 e 90,0 m, respectivamente. Em futuras
\end{abstract}

\footnotetext{
(1) Trabalho apresentado na XVI Reunião Brasileira de Manejo e Conservação do Solo e Água, em Aracaju (SE), de 23 a 27 de julho de 2006. Recebido para publicação em fevereiro de 2007 e aprovado em julho de 2007.

(2) Doutorando em Solos e Nutrição de Plantas do Departamento de Ciência do Solo, Universidade Federal de Lavras - UFLA. Caixa Postal 37, CEP 37200-000, Lavras (MG). Bolsista CNPq. E-mail: ivoneygontijo@yahoo.com.br.

(3) Professor adjunto do Departamento de Ciência do Solo, UFLA. Bolsista CNPq. E-mail: msouzadj@ufla.br

(4) Professor associado do Departamento de Ciências Exatas, UFLA. E-mail: marcelo.oliveira@ufla.br

(5) Mestrando em Solos e Nutrição de Plantas do Departamento de Ciência do Solo, UFLA. E-mail: cfaj@bol.com.br; piresbruno@passosuemg.br

(6) Aluno de Iniciação Científica. E-mail: carloemeoliveira@hotmail.com
} 


\begin{abstract}
amostragens para determinação da $\sigma_{p}$, em condições similares às da área estudada, sugere-se dispor os pontos de coleta com intervalo igual ao alcance de dependência espacial, visando associar menor esforço de amostragem com maior representatividade da área. Apesar de a razão de dependência espacial encontrada para as variáveis estudadas ter sido moderada, seu alcance deve ser considerado no planejamento de novas amostragens. A declividade do solo influenciou indiretamente os valores de $\sigma_{\mathrm{p}}$. $\mathrm{O}$ mapeamento da área permitiu observar zonas de maior e menor suscetibilidade à compactação, possibilitando a tomada de decisão sobre onde começar a trafegar para evitar danos à estrutura do solo.
\end{abstract}

Termos de indexação: compactação do solo, geoestatística, amostragem de solo.

\title{
SUMMARY: PLANNING THE SAMPLING FOR PRECONSOLIDATION PRESSURE OF A RED LATOSOL
}

\begin{abstract}
Preconsolidation pressure is currently used as a tool in the study of the soil compaction process. The purpose of this work was to determine the layout and number of samples required for the determination of the preconsolidation pressure $\left(\sigma_{p}\right)$ in a Red Latosol, as well as to characterize its variability and spatial distribution using classical statistics and geostatistics parameters. The study was carried out in an experimental farm area, in Lavras, Minas Gerais State, Brazil, conventionally tilled in the last 30 years. The experimental area was $32 \times 160 \mathrm{~m}\left(5.120 \mathrm{~m}^{2}\right)$ in a regular grid. Samples were collected at 68 equally spaced points $(10 \times 8 \mathrm{~m})$. Six transects were chosen: four transects with 4 points and two transects with 8 points were sampled to detect short distance variation. Ninetyeight undisturbed samples were collected at a depth of $0-3 \mathrm{~cm}$. The samples were subjected to the uniaxial compression test to establish $\sigma_{p}$ at field conditions. Using classical statistics parameters, the appropriate number of sampling points for $\sigma_{p}$ determination was 10 . The highest variability was obtained for $\sigma_{p}(C V 14.8 \%)$ and the lowest for water content (CV $12.0 \%)$. In both cases, the soil parameters were normally distributed, and the semivariograms were satisfactorily described by spherical models with a moderate spatial structure, and with spatial ranges of 19.5 and $90.0 \mathrm{~m}$, respectively. Under similar study conditions, intervals equal to the spatial range should be used for $\sigma_{p}$ determination in future studies in order to reduce the sampling effort and increase representativeness. Although the ratio of the spatial dependence of the studied variables was intermediate, the spatial range must be taken into consideration when planning sampling. Field slope influenced the $\sigma_{p}$ values directly. The constructed preconsolidation distribution map identified regions with high and low compaction susceptibility. These results will be useful to decide where machine traffic on the soil should begin as to avoid additional problems in the soil structure.
\end{abstract}

Index terms: soil compaction, geostatistics, soil sampling.

\section{INTRODUÇÃO}

Atualmente, um dos problemas que mais limitam a produção agrícola e a qualidade do ambiente em áreas intensamente mecanizadas é a compactação do solo. Assim, é necessário o conhecimento dos efeitos da compactação do solo para identificar estratégias de prevenção, com a finalidade de estabelecer metodologias para correta quantificação dos impactos causados em áreas agrícolas. A pressão de preconsolidação $\left(\sigma_{\mathrm{p}}\right)$ é definida como a maior pressão que o solo já suportou no passado (Dias Junior \& Pierce, 1996) e é uma medida da capacidade de suporte de carga do solo. A $\sigma_{p}$ é obtida a partir da curva de compressão do solo, que relaciona a densidade do solo (ou índice de vazios) com o logaritmo da pressão a ele aplicada. A aplicação no solo de pressões menores do que a pressão de preconsolidação causa deformações elásticas, portanto recuperáveis; já a aplicação de pressões maiores causa deformações plásticas, não recuperáveis (Holtz \& Kovacs, 1981). Essa propriedade tem sido utilizada por diversos autores como indicador de sustentabilidade da estrutura do solo em uma dada umidade e, ou, potencial matricial (Kondo \& Dias Junior, 1999; Imhoff et al., 2001; Silva et al., 2003; Oliveira et al., 2003; Dias Junior et al., 2005). No entanto, são escassos os estudos que consideram a variabilidade espacial de tal atributo. Assim, torna-se necessário um planejamento de amostragem eficiente e representativo para a $\sigma_{\mathrm{p}}$, em 
que se leve em consideração sua estrutura de dependência espacial. A estimativa do número adequado de amostras dos diversos atributos do solo, utilizando a Geoestatística como ferramenta de decisão,já foi abordada em alguns estudos (McBratney \& Webster, 1983; Oliveira, 1991; Souza Filho \& Oliveira, 1996; Souza et al., 1997; Gonçalves et al., 1999), visando minimizar os custos envolvidos, otimizando o processo de amostragem sem, no entanto, comprometer a sua confiabilidade.

Quando não se conhece o grau de autocorrelação espacial entre os pontos amostrais de determinada propriedade do solo, como no caso da estatística clássica, freqüentemente coleta-se uma quantidade excessiva de amostras para obter a precisão desejada. Assim, o conhecimento da dependência espacial das propriedades do solo é importante para nortear o processo de amostragem, promovendo redução nos custos de coleta.

Normalmente, pontos de amostragem localizados a pequenas distâncias são mais semelhantes entre si que pontos mais distantes (McBratney \& Webster, 1983). Na amostragem ao acaso, alguns pontos de amostragem podem ser tomados muito próximos uns dos outros, duplicando a informação e onerando o processo de amostragem. Assim, informações a respeito da continuidade da distribuição espacial entre as amostras, representada pelo alcance, permitirão a construção de conjuntos de dados independentes, possibilitando o uso da Estatística Clássica sem restrições. Além do mais, permite determinar onde se realizarão novas amostragens, se necessário; e a definição de novos esquemas de amostragem no campo, evitando uma situação de dependência espacial (Miller et al., 1988). Na literatura foram encontrados valores de alcance de $69 \mathrm{~m}$ para umidade do solo e de $22 \mathrm{~m}$ para densidade do solo em um Latossolo Vermelho eutroférrico (Souza et al., 2004). Libardi et al. (1986), entretanto, encontraram alcance de $16 \mathrm{~m}$ para umidade do solo em uma Terra Roxa Estruturada.

O objetivo deste trabalho foi determinar a configuração e o número adequado de amostras para determinação da $\sigma_{\mathrm{p}}$ em um Latossolo Vermelho distroférrico, bem como caracterizar sua variabilidade e distribuição espacial, utilizando métodos da Estatística Clássica e da Geoestatística.

\section{MATERIAL E MÉTODOS}

O experimento foi instalado na região do Sul de Minas, no município de Lavras-MG; a área encontrase a $21^{\circ} 14^{\prime} \mathrm{S}$ e $45^{\circ} 00$ ' W, a uma altitude de $918 \mathrm{~m}$. O clima da região, segundo a classificação de Köppen, é do tipo tropical úmido (Cwb), com duas estações bem definidas, inverno seco e verão chuvoso, e precipitação média anual de $1.593 \mathrm{~mm}$ (Brasil, 1992). O solo da área foi caracterizado como um Latossolo Vermelho distroférrico típico (LVdf) (Embrapa, 1999) textura muito argilosa (Quadro 1), apresentando relevo suave ondulado, localizado no ombro da encosta, com declividade média de $8,0 \%$. A área se encontrava sob plantio convencional nos últimos 30 anos, sendo submetida a uma aração e duas gradagens anualmente. Por ocasião da instalação do experimento, a área nesse ano não foi cultivada. Para evitar ocorrência de alterações na estrutura do solo pela ação das plantas daninhas, a área foi mantida livre destas por meio de capina manual alternada com aplicação de herbicida de pós-emergência (glyphosate).

Demarcou-se uma malha regular de $32 \times 160 \mathrm{~m}$ $\left(5.120 \mathrm{~m}^{2}\right)$, com 68 pontos distanciados a $10 \mathrm{~m}$ no eixo $\mathrm{X}$ e a $8 \mathrm{~m}$ no eixo $\mathrm{Y}$, mais seis transectos, sendo quatro com pontos distanciados de $2 \mathrm{~m}$ e dois com pontos distanciados de $1 \mathrm{~m}$, conforme figura 1 , visando detectar variações a pequenas distâncias, totalizando 98 amostras. A amostragem foi feita no mês de outubro de 2004, com o auxílio de um amostrador de Uhland, utilizando anéis de alumínio de $2,54 \mathrm{~cm}$ de altura por $6,30 \mathrm{~cm}$ de diâmetro, para obtenção de amostras indeformadas. Após coleta das amostras, estas foram cuidadosamente embaladas em filme plástico, parafinadas, para preservação da estrutura e manutenção da umidade natural, e posteriormente identificadas.

Em laboratório, cada amostra foi moldada segundo o volume do anel, sendo submetidas ao ensaio de compressão uniaxial (Bowles, 1986), trabalhando com sua umidade natural. As pressões aplicadas a cada amostra, utilizando um consolidômetro da marca Boart Longyear ${ }^{\circledR}$, obedeceram à seguinte ordem: 25 , $50,100,200,400,800$ e $1.600 \mathrm{kPa}$; cada pressão foi aplicada até que $90 \%$ da deformação máxima fosse alcançada (Holtz \& Kovacs, 1981), e somente então

\section{Quadro 1. Propriedades físico-hídricas do Latossolo Vermelho distroférrico típico (LVdf)}

\begin{tabular}{|c|c|c|c|c|c|c|c|c|c|}
\hline Profundidade & $\mathbf{K S A T}^{(1)}$ & VTP(2) & Macro $^{(3)}$ & $\operatorname{Micro}^{(3)}$ & $\operatorname{Ds}^{(4)}$ & $D p^{(5)}$ & Argila & Silte & Areia \\
\hline $\mathrm{cm}$ & $\mathrm{cm} \mathrm{h}^{-1}$ & 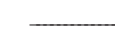 & $-\mathrm{m}^{3} \mathrm{~m}^{-3}$ & 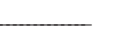 & $-\mathrm{k}$ & $-3+$ & 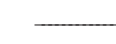 & $\mathrm{g} \mathrm{kg}^{-1}$ & - \\
\hline $0-3$ & 9,48 & 0,59 & 0,19 & 0,40 & 1,14 & 2,79 & 630 & 176 & 194 \\
\hline
\end{tabular}


aplicou-se nova pressão. Após o término do ensaio, as amostras foram levadas à estufa a $105-110^{\circ} \mathrm{C}$, até massa constante, para determinação da umidade gravimétrica, conforme Embrapa (1997).

Realizado o ensaio, a curva de compressão do solo foi obtida, plotando-se a pressão aplicada no eixo das abscissas, em escala logarítmica, versus a densidade do solo (Ds) correspondente no eixo das ordenadas, em escala decimal, conforme Dias Junior \& Pierce (1996).

A partir das curvas de compressão foram obtidas as $\sigma_{\mathrm{p}}$, determinadas de acordo com Dias Junior \& Pierce (1995).

Inicialmente foi realizada a análise exploratória dos dados por meio da Estatística Descritiva, obtendose as seguintes medidas: média aritmética, mediana, variância amostral, desvio-padrão, coeficiente de variação, valores máximo e mínimo, amplitude e coeficientes de assimetria e de curtose.

A definição do número de pontos amostrais (n) que representa os atributos do solo foi feita com base na equação (Cline, 1944):

$$
n=\left(\frac{t \alpha / 2 \cdot C V}{e r}\right)^{2}
$$

em que t $\alpha$ : valor da tabela de distribuição de Student para o nível de probabilidade $\alpha / 2$ (bilateral); CV: coeficiente de variação (\%); e er: erro relativo admitido em torno da média (\%).

A análise de distribuição de freqüência dos dados foi realizada para verificar sua normalidade, utilizando o teste de Shapiro-Wilk a $5 \%$. Essa análise é importante, pois, para utilização da equação 1 , exigese que a distribuição dos resultados seja normal, além de que eles sejam espacialmente independentes.

A análise da dependência espacial foi feita pela Geoestatística, com auxílio do software GS+ (Robertson, 1998), que realiza os cálculos das semivariâncias amostrais, cuja expressão pode ser encontrada em Vieira et al. (1983):

$$
\hat{\gamma}(h)=\frac{\sum_{i=1}^{n(h)}[z(x i)-z(x i+h)]^{2}}{2 n(h)}
$$

em que $n(h)$ é o número de pares amostrais $[z(x 1)$; $z(x i+h)]$ separados pelo vetor $h$, sendo $z(x i)$ e $z(x i+h)$ os valores numéricos observados do atributo analisado, para dois pontos $x i$ e $x i+h$ separados pelo vetor $h$. $O$ semivariograma é representado pelo gráfico de $\gamma(h)$ versus $h$. Após o ajuste de um modelo matemático aos valores calculados de $\gamma(h)$, são definidos os parâmetros do modelo teórico para o semivariograma (efeito pepita, Co; alcance da dependência espacial, a; e patamar, C). Amostras separadas por distâncias menores que o alcance são espacialmente correlacionadas, ao passo que as separadas por distâncias maiores não o são.

Foi calculada a razão de dependência espacial $(R D)$, que é a proporção em percentagem do efeito pepita (Co) em relação ao patamar $(\mathrm{Co}+\mathrm{C})$ - equação 3 , que, de acordo com Cambardella et al. (1994), apresenta a seguinte proporção: (a) dependência forte, $\leq 25 \%$; (b) dependência moderada, de 25 a $75 \%$; e (c) dependência fraca, $>75 \%$.

$$
R D=\left(\frac{C o}{C o+C}\right) * 100
$$

$\mathrm{Na}$ determinação da existência ou não de dependência espacial, utilizou-se o exame de semivariogramas, por meio do programa GS+ (Robertson, 1998). Em caso de dúvida entre mais de um modelo para o mesmo semivariograma, utilizouse a técnica de validação conhecida como jack-knifing, a qual consiste em retirar, individualmente, cada ponto medido da área estudada, e o seu valor é estimado pelo modelo como se ele nunca existisse.

\section{RESULTADOS E DISCUSSÃO}

Os resultados referentes à análise descritiva para $\sigma_{\mathrm{p}}$ e umidade do solo são apresentados no quadro 2 . Os valores da média e da mediana, para ambos os atributos do solo, estão próximos, mostrando haver distribuição simétrica, o que pode ser confirmado pelos valores de assimetria próximos de zero. Os resultados referentes ao teste de Shapiro-Wilk a $5 \%(\mathrm{P}>0,05)$ reforçam a aceitação da hipótese de normalidade dos erros (Quadro 2). O conhecimento da distribuição de freqüência dos dados de uma variável tem importantes conseqüências, pois a análise da variância e os testes de significância normalmente usados na Estatística Clássica (Tukey, F, t, etc.) baseiam-se na distribuição

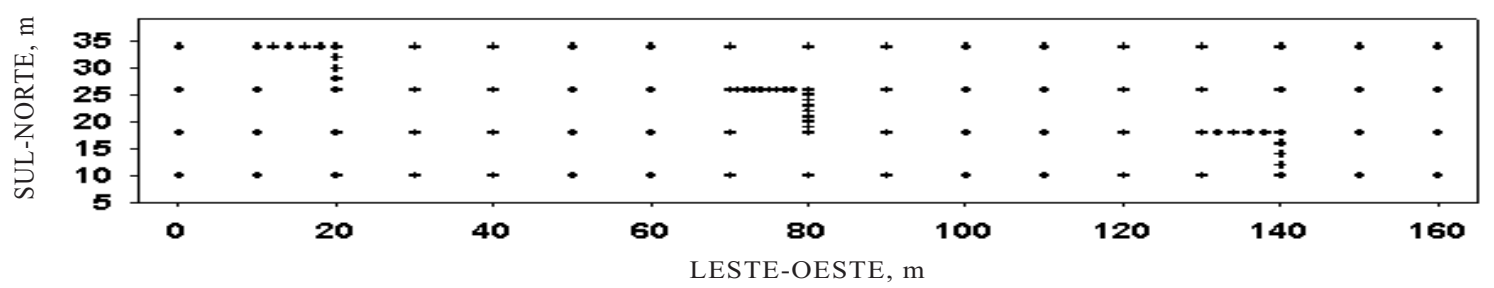

Figura 1. Esquema de amostragem realizada na área experimental. 
Quadro 2. Resumo estatístico dos dados de pressão de preconsolidação $\left(\sigma_{p}\right)$ e umidade do solo $(U)$ obtidos a partir de 98 amostras

\begin{tabular}{lcc}
\hline Parâmetro estatístico & $\sigma \mathbf{p}$ & $\mathbf{U}$ \\
\hline & $\mathrm{kPa}$ & $\mathrm{kg} \mathrm{kg}{ }^{-1}$ \\
Média & 341,9 & 0,2180 \\
Mediana & 334,7 & 0,2160 \\
Variância amostral & 2543,8 & 0,00071 \\
Desvio-padrão & 50,4 & 0,027 \\
CV (\%) & 14,8 & 12,0 \\
Mínimo & 220,8 & 0,1615 \\
Máximo & 469,0 & 0,2798 \\
Amplitude & 248,2 & 0,1183 \\
Assimetria & 0,20 & 0,10 \\
Curtose & $-0,63$ & $-0,62$ \\
P valor, teste de Shapiro Wilk & $0,2427^{(1)}$ & $0,4748(1)$ \\
\hline
\end{tabular}

${ }^{(1)}$ Possui distribuição normal pelo teste Shapiro-Wilk a $5 \%$.

normal. Entretanto, esse fato não deve ser motivo de maiores preocupações quando se aborda somente a Geoestatística (Clark, 1979).

Observa-se que os valores de $\sigma_{\mathrm{p}}$ apresentaram elevada amplitude, 248,2 $\mathrm{kPa}$ (Quadro 2 ), com valores mínimo e máximo de 220,8 e 469,0 kPa, respectivamente. A elevada amplitude da $\sigma_{\mathrm{p}}$ reflete o manejo empregado na área, já que o tráfego de máquinas ocorre aleatoriamente, além da constante alteração da estrutura do solo pelas operações de cultivo convencional, como o manejo das plantas daninhas, realizado por meio de capina manual alternada com aplicação de herbicida de pós-emergência, o que promove a inversão das camadas e deposição diferencial de partículas, influenciando a $\sigma_{\mathrm{p}}$. Ressalta-se que o valor mínimo está acima das pressões médias aplicadas pelo maquinário agrícola, as quais se situam entre 100 e $200 \mathrm{kPa}$ (Carpenedo, 1994). Castro Neto (2001), trabalhando com trator MF $299^{\circledR}$, concluiu que este aplicou uma pressão de $116,9 \mathrm{kPa}$ ao solo. Desse modo, pressões menores que a $\sigma_{\mathrm{p}}$ mínima $(220,8 \mathrm{kPa})$ não causariam problemas adicionais à estrutura do solo na área estudada, na presente condição de umidade. No entanto, é necessário considerar que os valores da umidade do solo são relativamente baixos e que normalmente podem ocorrer valores mais altos durante o ano, modificando o comportamento da $\sigma_{\mathrm{p}}$.

A $\sigma_{\mathrm{p}}$ e umidade do solo apresentaram coeficientes de variação de 14,8 e $12,0 \%$, respectivamente valores considerados médios, de acordo com Warrick $\&$ Nielsen (1980) - corroborando os resultados obtidos por Kondo (2003). Esse índice é de grande importância para definição do número adequado de amostras a serem coletadas para se obter um valor representativo
(Melo Filho et al., 2006) de determinado atributo do solo. Entretanto, como uma das pressuposições para utilização da equação 1 é a independência dos dados, optou-se por selecionar pontos na malha amostral (Figura 1) que apresentassem total independência espacial, ou seja, pontos separados por distâncias maiores que o alcance. Desse modo, o novo valor do coeficiente de variação para a $\sigma_{\mathrm{p}}$ foi de $16,29 \%$. Os pontos foram selecionados da seguinte maneira: tomou-se aleatoriamente o primeiro ponto e, em seguida, respeitou-se a distância do alcance, que foi de 19,5 m para pressão de preconsolidação (Quadro 3), para seleção dos demais pontos, totalizando 18 pontos selecionados.

O número de amostras para obter um valor médio representativo da $\sigma_{\mathrm{p}}$, para um nível de confiança desejado, pode ser calculado pela equação 1 . A figura 2 mostra o número necessário de amostras em nível de significância de $5 \%$, para variações em torno da média, medida pelo erro relativo, de 5 a $30 \%$. O número de amostras necessário para obter variação de $10 \%$ em torno da média, com um nível de significância a $5 \%$, seria de 10 pontos amostrais nas condições estudadas (Figura 2). Para variação de $5 \%$ em torno da média, esse número seria de 42 - valor considerado alto do ponto de vista prático. Há aumento no número de amostras à medida que se reduz a variação em torno da média. Desse modo, o aumento da exatidão da estimativa da média está associado ao acréscimo considerável do esforço, onerando o processo de amostragem sem incremento proporcional em precisão.

Os coeficientes de assimetria e de curtose são apresentados para efeito de comparação com a distribuição normal (Figura 3), sendo eles próximos de zero, o que está de acordo com os valores de CV, que foram de 14,8 e 12,0\% para $\sigma_{p}$ e umidade do solo, respectivamente.

De acordo com o teste de Shapiro-Wilk a $5 \%$ (Quadro 2), há normalidade nos atributos estudados, como pode ser visualizado na figura 3. Segundo Isaaks \& Srivastava (1989), mais importante que a normalidade dos dados é a ocorrência ou não do chamado efeito proporcional, ou seja, que a média e a

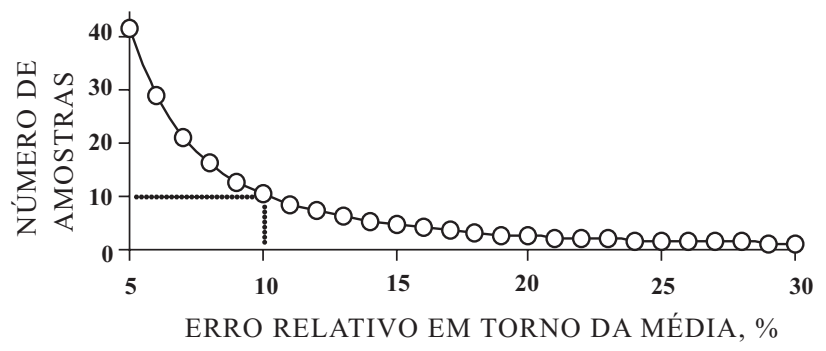

Figura 2. Número necessário de amostras para estimativa da média da $\sigma_{\mathrm{p}}$ conforme o erro relativo em torno da média, em nível de significância de $5 \%$. 
variabilidade dos dados sejam constantes na área de estudo, fato esse observado na figura 4, ocorrendo assim a estacionaridade isotrópica - fato essencial na aplicação da Geoestatística. Não é possível visualizar se há ou não anisotropia dos dados devido à configuração da área, que possui formato retangular com predomínio de pontos em uma direção (leste-oeste). Desse modo, assumiu-se por hipótese de trabalho a isotropia dos dados para construção do semivariograma e posterior krigagem.

O comportamento dos dados em relação às direções da malha amostral é mostrado na figura 4, na qual se nota que os dados estão distribuídos homogeneamente em toda a área, não havendo regiões com concentração de valores altos ou baixos em zonas específicas da área estudada. Essa análise indica não haver tendência nas direções da malha amostral, o que significa não existirem, também, maiores problemas ao assumir a hipótese de estacionaridade isotrópica dos dados (Queiroz et al., 1999).
Dentre os parâmetros obtidos pela Estatística Espacial está o alcance da dependência espacial, que representa a distância em que os pontos amostrais estão correlacionados entre si (Vieira, 2000). Dessa forma, amostras localizadas numa área de raio igual ao alcance são mais homogêneas entre si do que com aquelas localizadas fora dessa área. Portanto, o conhecimento do alcance da dependência espacial permitirá a definição desse intervalo, que deverá ser, no mínimo, igual ao alcance, garantindo a independência entre os pontos amostrais, diminuindo o esforço e os custos de amostragem, não comprometendo sua representatividade.

O modelo teórico ajustado às duas variáveis estudadas foi o esférico (Quadro 3), que é bastante utilizado por vários pesquisadores para descrever o comportamento de semivariogramas de atributo de solo e de plantas (Salviano et al., 1998).

O efeito pepita ( $\mathrm{Co}$ ) é um parâmetro do semivariograma que indica variabilidade não
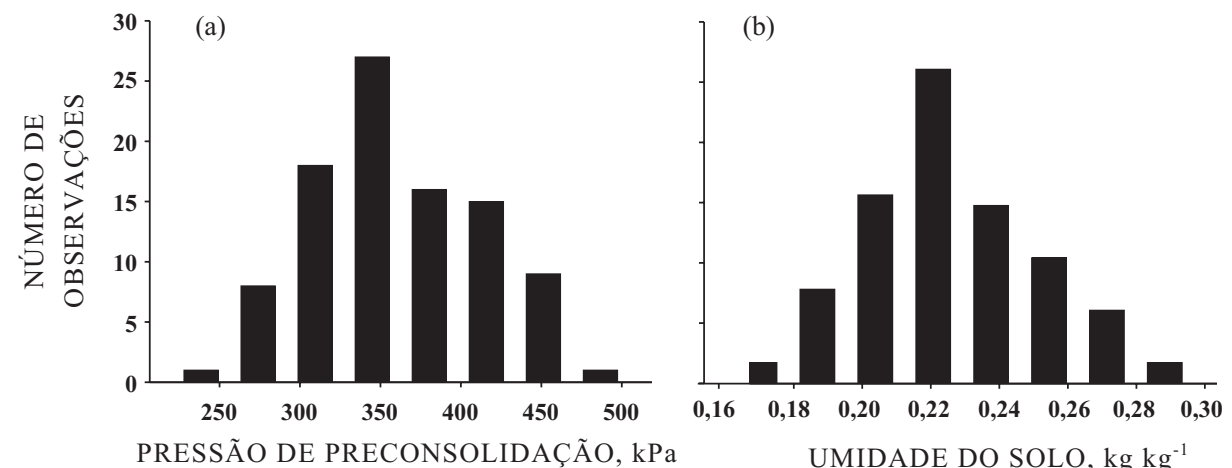

Figura 3. Distribuição de freqüência para as 98 amostras de pressão de preconsolidação (a) e umidade do solo (b).
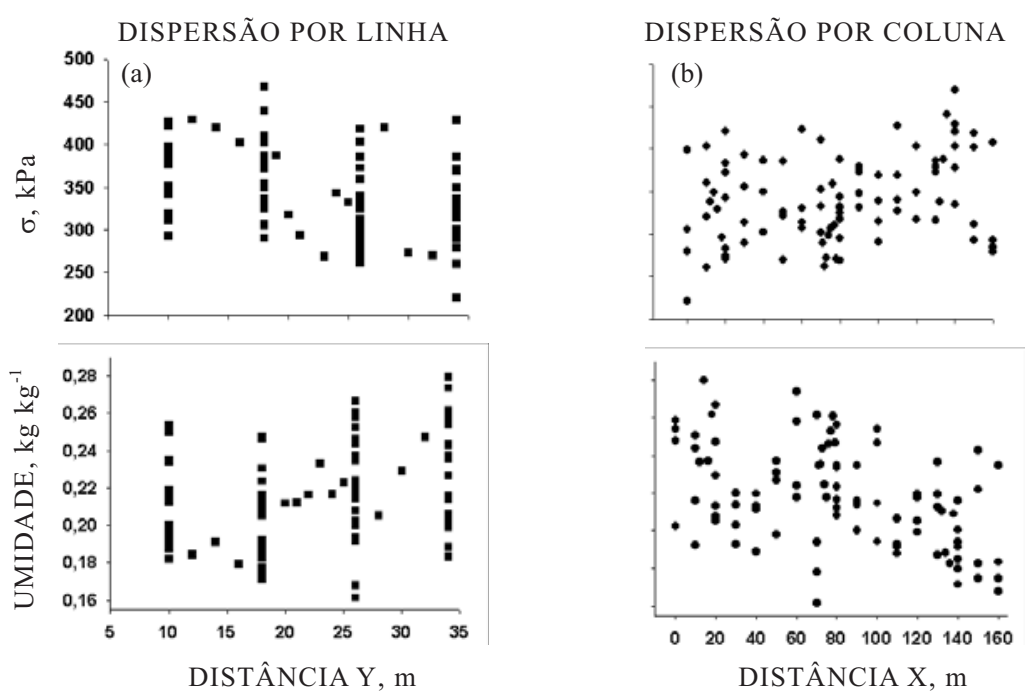

Figura 4. Gráficos de dispersão dos dados de pressão de preconsolidação $\left(\sigma_{\mathrm{p}}\right)$ e umidade do solo, por linha (a) e coluna (b). 
explicada, que pode ser devida a erros de medição ou variação não detectada pela escala de amostragem (Cambardella et al., 1994), além de ser influenciada pelas características extrínsecas ao solo ocasionadas pela ação antrópica. Esse parâmetro pode ser quantificado pela razão entre o efeito pepita e o patamar $(\mathrm{Co}+\mathrm{C})$, chamada de razão da dependência espacial (RD), proposta por Cambardella et al. (1994). Pelo quadro 3, observa-se que a RD foi praticamente a mesma para as duas variáveis estudadas, sendo considerada uma dependência moderada (entre $25 \mathrm{e}$ $75 \%$ ), fato explicado pelas variações extrínsecas causadas pelo preparo do solo (Cambardella et al., 1994), que influenciam sobremaneira ambas as variáveis, principalmente a $\sigma_{p}$, por meio do tipo, da intensidade e da freqüência da carga aplicada ao solo (Horn, 1988; Lebert \& Horn, 1991).

Os semivariogramas experimentais, com os respectivos modelos ajustados, são apresentados na figura 5. Os alcances de 19,5 e 90,0 m para $\sigma_{\mathrm{p}}$ e umidade do solo, respectivamente, indicam a amplitude de correlação espacial entre as observações de cada variável, representando o raio de um círculo no qual as amostras têm correlação entre si. Portanto, não se recomenda amostrar mais de um ponto dentro desse intervalo, sob pena de haver duplicidade de amostragem, bem como alto ônus para 0 agricultor.

Se as amostras forem coletadas seguindo a presente recomendação de amostragem para a área em estudo (10 pontos distanciados de, no mínimo, 19,5 m), será possível aplicar somente a Estatística Clássica e seus parâmetros, sendo inviabilizado, portanto, o uso da Geoestatística e suas aplicações (Quadro 4). Isso se deve ao fato de que as amostras futuramente coletadas estarão dispostas a distâncias maiores que o alcance espacial, proporcionando independência total entre elas.

Com os parâmetros do modelo ajustado ao semivariograma, utilizando-se o processo de krigagem ordinária pontual com 16 vizinhos, foram estimados os valores da $\sigma_{\mathrm{p}}$ e umidade do solo na área estudada. A partir dos valores estimados, construíram-se os mapas de isolinhas apresentados na figura 6 . A área possui declividade média de $8,0 \%$ no sentido sul-norte (Figura 6), que influencia diretamente os valores de umidade do solo, que por sua vez influenciam os valores da $\sigma_{p}$, corroborando os resultados de Ferrero et al. (2005). A correlação da declividade, tanto com a umidade quanto com a $\sigma_{\mathrm{p}}$, foi significativa a $5 \%$, com coeficiente de correlação de 0,32 para ambas as variáveis. Devido a esse fato, são observados maiores valores de umidade na região norte, principalmente mais a leste da área, os quais foram maiores que $0,2150 \mathrm{~kg} \mathrm{~kg}^{-1}$ (Figura 6); essa região apresenta menor capacidade de suporte de carga, sendo considerada, portanto, mais suscetível à compactação do solo.
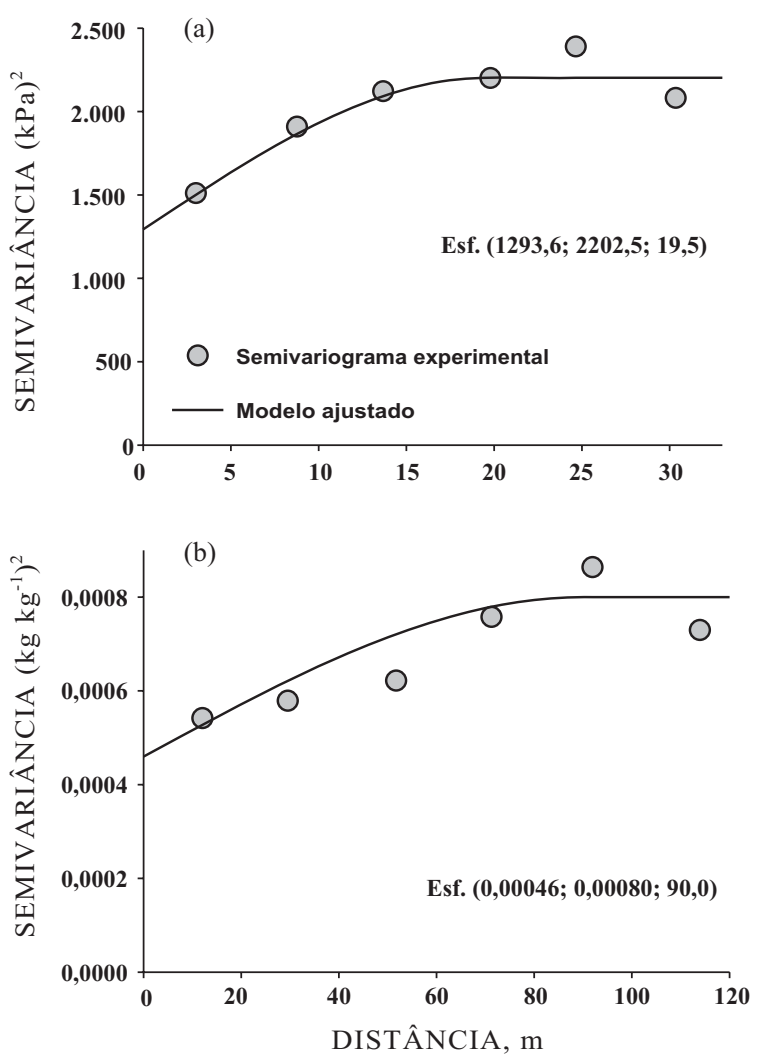

Figura 5. Semivariograma da pressão de preconsolidação (a) e umidade do solo (b). Valores entre parênteses são efeito pepita $(\mathrm{Co})$, patamar $($ Co $+C)$ e alcance $(\mathbf{a})$, respectivamente.

Quadro 3. Estimativa dos parâmetros do modelo teórico ajustado para pressão de preconsolidação $\left(\sigma_{\mathrm{p}}\right)$ e umidade do solo (U)

\begin{tabular}{|c|c|c|c|c|c|c|}
\hline Variável & Modelo & Co & $\mathrm{Co}+\mathrm{C}$ & Alcance & $\mathbf{R}^{2}$ & RD \\
\hline & & & & $\mathrm{m}$ & & $\%$ \\
\hline$\sigma \mathbf{p}(\mathrm{kPa})$ & esférico & 1293,6 & 2202,5 & 19,5 & 0,88 & 59 \\
\hline $\mathrm{U}\left(\mathrm{kg} \mathrm{kg}^{-1}\right)$ & esférico & 0,00046 & 0,00080 & 90,0 & 0,80 & 58 \\
\hline
\end{tabular}

Co: efeito pepita, Co + C: patamar, $R^{2}$ : coeficiente de determinação, RD: razão da dependência espacial [Co/(Co + C) 100]. 


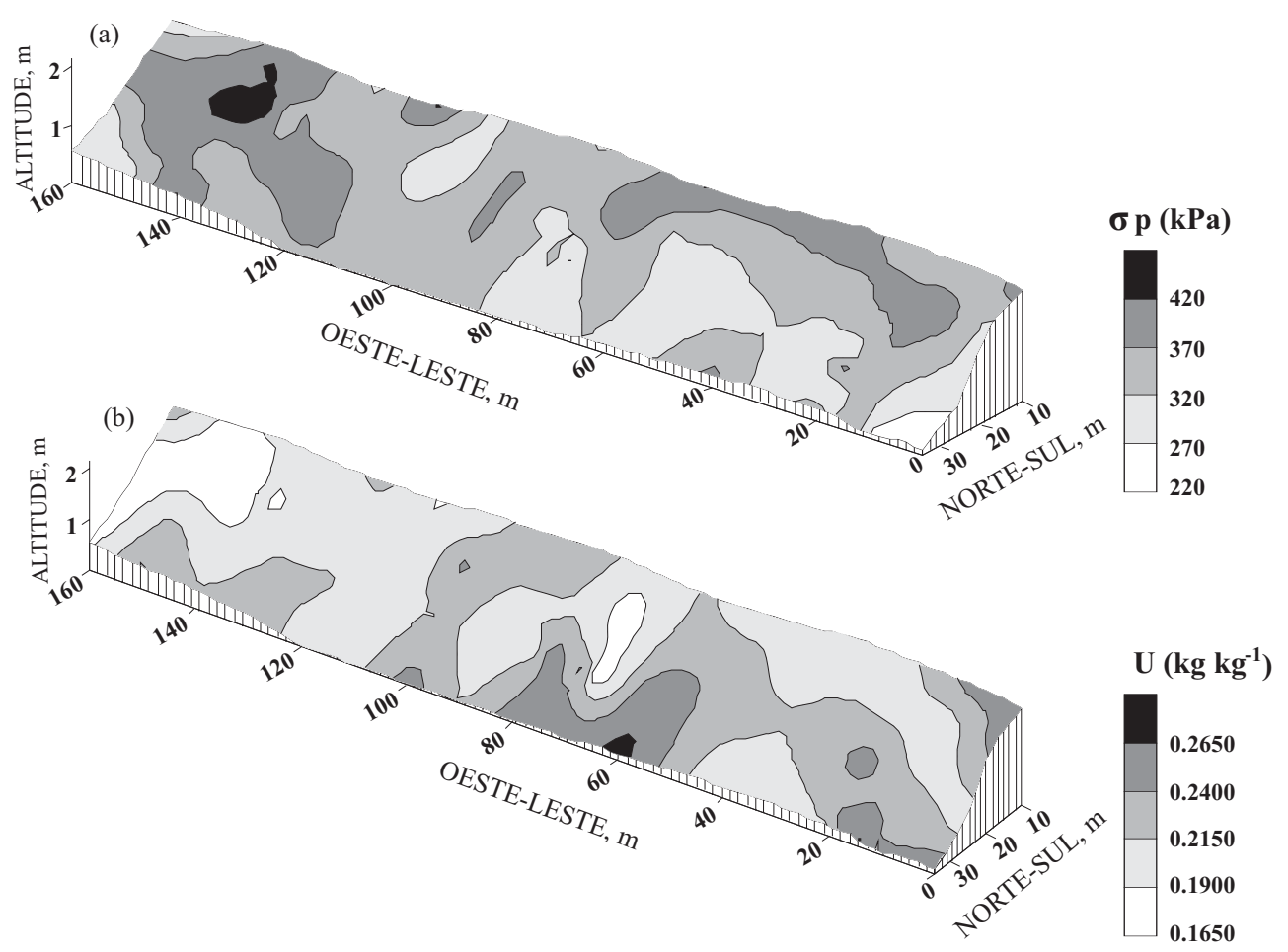

Figura 6. Mapa de isolinhas da pressão de preconsolidação (a) e umidade do solo (b), representado conjuntamente com o relevo da área.

Quadro 4. Possibilidades de uso da Estatística Clássica e Geoestatística para a amostragem recomendada (pontos independentes)

\begin{tabular}{lll}
\hline & & Método \\
\cline { 3 - 3 } Objetivo para amostragem & Estatística clássica & Geoestatística \\
\hline Média aritmética & Sim & Não \\
Desvio-padrão & Sim & Não \\
Curtose & Sim & Não \\
Assimetria & Sim & Não \\
Coeficiente de variação & Sim & Não \\
Teste de normalidade & Sim & Não \\
Intervalo de confiança & Sim & Não \\
Mapeamento (Krigagem) & Não & Não \\
Média espacial & Não & Não \\
\hline
\end{tabular}

Nota-se que a oeste da área existe uma região que apresentou valores de $\sigma_{\mathrm{p}}$ acima de $370 \mathrm{kPa}$, a qual está situada na lateral da lavoura, próxima aos carreadores, com índices maiores de compactação, devido ao tráfego intenso de máquinas, conforme verificado por Silva et al. (2004). Por outro lado, essa região apresenta maior capacidade de suporte de carga, podendo ser, portanto, trafegada sem que haja compactação adicional do solo. Outro fato que contribuiu para elevada $\sigma_{\mathrm{p}}$, nessa região, foi a baixa umidade $\left(<0,2150 \mathrm{~kg} \mathrm{~kg}^{-1}\right)$, influenciada pelas maiores cotas de altitude. Entretanto, uma maior capacidade de suporte de carga pode limitar o desenvolvimento do sistema radicular das culturas, devido à alta pressão que as raízes têm de realizar para se desenvolverem, visto que as pressões exercidas por elas têm de ser iguais às de $\sigma_{p}$ (Römkens \& Miller, 1971). 
O mapa de isolinhas pode, portanto, ser usado como ferramenta para auxiliar na tomada de decisão de onde e quando trafegar em uma determinada área, optandose por trafegar inicialmente em regiões menos suscetíveis à compactação do solo, em razão dos maiores valores de $\sigma_{p}$, preservando em conseqüência a estrutura do solo.

Apesar da moderada dependência espacial encontrada para a $\sigma_{p}$, seu alcance deve ser considerado no planejamento de novas amostragens. Assim, em futuras amostragens para determinação da pressão de preconsolidação, em condições similares às da área estudada, sugere-se dispor os pontos de coleta (10 pontos) com intervalo, no mínimo, igual ao alcance de dependência espacial (19,5 m), associando um menor esforço de amostragem com uma maior representatividade da área.

\section{CONCLUSÕES}

1. A declividade influenciou indiretamente a pressão de preconsolidação, por meio da umidade do solo.

2. A pressão de preconsolidação e a umidade do solo apresentaram moderada estrutura de dependência espacial, com alcance de 19,5 e 90,0 m, respectivamente.

3. O mapeamento da área permitiu observar zonas de maior e menor suscetibilidade à compactação, possibilitando a tomada de decisão sobre onde começar a trafegar sem causar problemas adicionais à estrutura do solo.

4. O conhecimento da distribuição espacial da pressão de preconsolidação e da umidade do solo pode ser usado para desenvolvimento de estratégias de manejo que minimizem os riscos da compactação adicional do solo e os impactos causados pelas operações motomecanizadas.

\section{AGRADECIMENTOS}

Ao Dr. Donald Nielsen, professor da University of California, pelas críticas e sugestões para o aprimoramento deste trabalho; e ao CNPq, pela concessão de bolsa ao primeiro autor.

\section{LITERATURA CITADA}

BOWLES, J.E. Engineering properties of soils and their measurements. 3.ed. New York, McGraw-Hill, 1986. 218p.

BRASIL. Ministério da Agricultura e Reforma Agrária. Normais climatológicos (1961-1990). Brasília, Secretaria Nacional de Irrigação, Departamento Nacional de Meteorologia, 1992. 84p.
CAMBARDELLA, C.A.; MOORMAN, T.B.; NOVAK, J.M.; PARKIN, T.B.; KARLEN, D.L.; TURCO, R.F. \& KONOPKA, A.E. Field-scale variability of soil properties in central Iowa soils. Soil Sci. Soc.Am. J., 58:1501-1511, 1994.

CLARK, I. Practical geostatistics. London, Applied Science Publishers, 1979. 128p.

CARPENEDO, V. Compressibilidade de solos em sistemas de manejo. Porto Alegre, Universidade Federal do Rio Grande do Sul, 1994. 106p. (Tese de Doutorado).

CASTRO NETO, P. Desenvolvimento e avaliação de equipamentos e metodologia para determinação de parâmetros físicos do solo relacionados a dias trabalháveis com máquinas agrícolas. Botucatu, Universidade Estadual Paulista, 2001. 155p.(Tese de Doutorado).

CLINE, M.G. Principles of soil sampling. Soil Sci., 58:275-288, 1944.

DIAS JUNIOR, M.S. \& PIERCE, F.J. A simple procedure for estimating preconsolidation pressure from soil compression curves. Soil Technol., 8:139-151, 1995.

DIAS JUNIOR, M.S. \& PIERCE, F.J. O processo de compactação do solo e sua modelagem. R. Bras. Ci. Solo, 20:175-182, 1996.

DIAS JUNIOR, M.S.; LEITE, F.P.; LASMAR JÚNIOR, E. \& ARAUJO JUNIOR, C.F. Traffic effects on the soil preconsolidation pressure due to Eucalyptus harvest operations. Sci. Agric., 62:248-255, 2005.

EMPRESA BRASILEIRA DE PESQUISA AGROPECUÁRIA EMBRAPA. Centro Nacional de Pesquisas de Solos. Manual de métodos de análise de solo. Rio de Janeiro, 1997. 212p.

EMPRESA BRASILEIRA DE PESQUISA AGROPECUÁRIA EMBRAPA. Centro Nacional de Pesquisas de Solos. Sistema brasileiro de classificação de solos. Rio de Janeiro, 1999. 412p.

FERRERO, A.; USOWICZ, B. \& LIPIEC, J. Effects of tractor traffic on spatial variability of soil strength and water content in grass covered and cultivated sloping vineyard. Soil Till. Res., 84:127-138, 2005.

GONÇALVES, A.C.A.; FOLEGATTI, M.V. \& VIEIRA, S.R. Padrões de amostragem e intensidade de krigagem na caracterização do armazenamento de água no solo, em área irrigada por pivô central. R. Bras. Ci. Solo, 23:485495, 1999.

HOLTZ, R.D. \& KOVACS, W.D. An introdution to geotechnical engineering. Englewood Cliffs, Prentice-Hall, 1981. 733p.

HORN, R. Compressibility of arable land. Catena, 11:53-71, 1988. Suplemento

IMHOFF, S.; SILVA, A.P.; DIAS JUNIOR, M.S. \& TORMENA, C.A. Quantificação de pressões críticas para o crescimento das plantas. R. Bras. Ci. Solo, 25:11-18, 2001.

ISAAKS, E.H. \& SRIVASTAVA, R.M. An introdution to applied geostatistics. New York, Oxford University Press, 1989. $561 \mathrm{p}$. 
LEBERT, M. \& HORN, R. A method to predict the mechanical strengh of agricultural soils. Soil Till. Res., 19:274-286, 1991.

LIBARDI, P.L.; PREVEDELLO, C.L.; PAULETTO, E.A. \& MORAES, S.O. Variabilidade espacial da umidade, textura e densidade de partículas ao longo de uma transeção. R. Bras. Ci. Solo, 10:85-90, 1986.

KONDO, M.K. Variabilidade espacial do comportamento compressivo do solo e mapas de trafegabilidade na cultura do cafeeiro irrigado. Lavras, Universidade Federal de Lavras, 2003. 166p. (Tese de Doutorado)

KONDO, M.K. \& DIAS JUNIOR, M.S. Compressibilidade de três Latossolos em função da umidade e uso. R. Bras. Ci. Solo, 23:211-218, 1999.

McBRATNEY, A.B. \& WEBSTER, R. How many observations are needed for regional estimation of soil properties? Soil Sci., 135:177-183, 1983.

MELO FILHO, J.F.; OLIVEIRA, A.S.; LOPES, L. \& VELLAME, L.M. Análise exploratória e variabilidade da densidade do solo em um perfil de Latossolo Amarelo Coeso dos tabuleiros costeiros da Bahia. Ci. Agrotec., 30:199-205, 2006.

MILLER, M.P.; SINGER, M.J. \& NIELSEN, D.R. Spatial variability of wheat yield and soil properties on complex hills. Soil Sci. Soc. Am. J., 52:1133-1141, 1988.

OLIVEIRA, G.C.; DIAS JUNIOR, M.S.; CURI, N. \& RESCK, D.V.S. Compressibilidade de um Latossolo Vermelho argiloso de acordo com a tensão de água no solo, uso e manejo. R. Bras. Ci. Solo, 27:773-781, 2003.

OLIVEIRA M.S. Planos amostrais para variáveis espaciais utilizando Geoestatística. Campinas, Universidade Estadual de Campinas, 1991. 110p. (Tese de Mestrado)

QUEIROZ, J.E.; CRUCIANI, D.E. \& LIBARDI, P.L. Variabilidade espacial da porosidade drenável de um solo de várzea, no município de Piracicaba, Estado de São Paulo. R. Bras. Eng. Agric. Amb., 3:135-139, 1999.
ROBERTSON, G.P. GS: Geostatistics for the environmental sciences (version 5.1 for windows). Plainwell, Gamma Design Software, 1998. 152p.

RÖMKENS, M.J.M. \& MILLER, R.R. Predicting root size and frequency from one-dimensional consolidation data: A mathematical model. Plant Soil, 35:237-248, 1971.

SALVIANO, A.A.C.; VIEIRA, S.R. \& SPAROVEK, G. Variabilidade espacial de atributos de solo e de Crotalaria juncea L. em área severamente erodida. R. Bras. Ci. Solo, 22:115-122, 1998.

SILVA, R.B.; DIAS JUNIOR, M.S.; SILVA, F.A.M. \& FOLE, S.M.O. O tráfego de máquinas agrícolas e as propriedades físicas, hídricas e mecânicas de um Latossolo dos cerrados. R. Bras. Ci. Solo, 27:211-218, 2003.

SILVA, V.R.; REICHERT, J.M. \& REINERT, D.J. Variabilidade espacial da resistência do solo à penetração em plantio direto. Ci. Rural, 34:399-406, 2004.

SOUZA FILHO, M.D.I. \& OLIVEIRA, M.S. Planejamento da amostragem de solos visando à estimação da média espacial do teor de cálcio utilizando a geoestatística. Ci. Agrotec., 20:387-393, 1996.

SOUZA, L.; COGO, N.P. \& VIEIRA, S.R. Variabilidade de propriedades físicas e químicas do solo em um pomar cítrico. R. Bras. Ci. Solo, 21:367-372, 1997.

SOUZA, Z.M.; MARQUES JÚNIOR, J. \& PEREIRA, G.T. Variabilidade espacial de atributos físicos do solo em diferentes formas do relevo sob cultivo de cana-de-açúcar. R. Bras. Ci. Solo, 28:937-944, 2004.

VIEIRA, S.R. Geoestatística em estudos de variabilidade espacial do solo. In: NOVAIS, R.F.; ALVAREZ V., V.H. \& SCHAEFER, G.R., eds. Tópicos em ciência do solo. Viçosa, MG, Sociedade Brasileira de Ciência do Solo, 2000. v.1. p.1-54.

VIEIRA, S.R.; HATFIEL, J.L.; NIELSEN, D.R. \& BIGGAR, J.W. Geoestatistical theory and application to variability of some agronomical properties. Hilgardia, 51:1-75, 1983.

WARRICK, A.W. \& NIELSEN, D.R. Spatial variability of soil physical properties in the field. In: HILLEL, D., ed. Applications of soil physics. New York, Academic Press,1980. p.319-344. 